\title{
Precision Medicine: Omics-tailored Clinics
}

\author{
Saliha Ece Acuner-Ozbabacan \\ Department of Bioengineering, Istanbul Medeniyet University, Istanbul 34700, Turkey \\ *Corresponding author - ece.ozbabacan@medeniyet.edu.tr
}

\begin{abstract}
We are now in a data-driven biomedical era in which the aim of precision medicine is to collect, investigate and interpret the omics data effectively and integratively to be able to implement the results in healthcare with high accuracy. Recent promising applications show that molecularly classifying a disease into subpopulations is beneficial for both patients and drug developers.
\end{abstract}

Keywords: precision medicine, personalized medicine, genome sequencing, proteome, bioinformatics.

\section{The concept of personalized/precision medicine}

Precision medicine is a patient-specific approach targeting to tailor the disease treatment based on the personal genetic information as well as clinical data, environmental parameters and the patient's lifestyle; as opposed to the conventional generic approach considering the average person ${ }^{[1]}$. This concept, especially "personalized medicine", has been misinterpreted as suggesting unique treatments for individual patients. However, the aim is rather to be able to choose more accurate treatment strategies for subpopulations that share commonalities in their particular disease susceptibilities or responses to specific treatments ${ }^{[1]}$. The meanings of "precision medicine" and "personalized medicine" terms significantly overlap and they have been used interchangeably but the National Research Council preferred to use the relatively newer "precision medicine" term in their 2011 report, due to the higher risk of misinterpreting the latter term ${ }^{[2]}$. This report was on developing a framework to use the ever-increasing molecular data of individual patients to improve health outcomes ${ }^{[2]}$. The interest in precision medicine made another peak after US President Obama announced the launch of the Precision Medicine Initiative in his State of the Union address in January 2015 ${ }^{[3]}$ (Figure 1). Personalized/precision medicine, having the potential of being diagnostic, predictive, therapeutic and preventative, was envisioned to be the future of healthcare 15 years ago ${ }^{[4]}$ and today we started to live in that future.
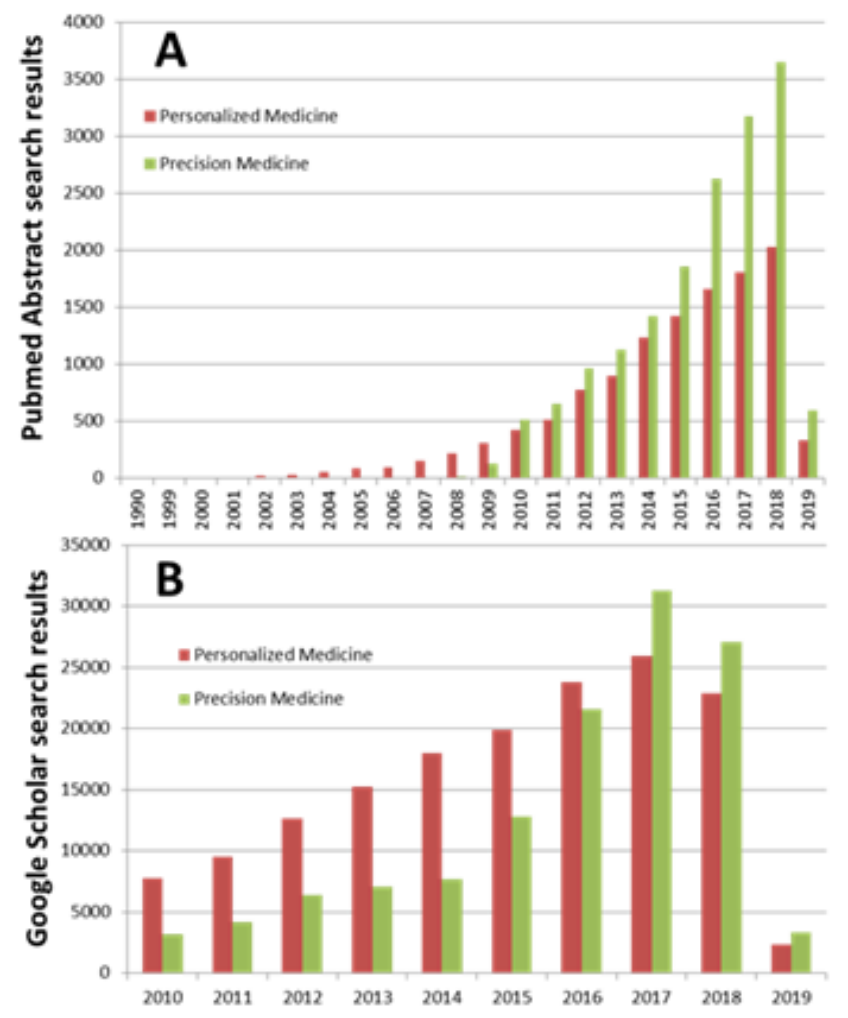

Figure 1: The changes in the number of publications using the terms "Personalized Medicine" and "Precision Medicine" over the years. A. number of abstracts in PubMed (based on the data retrieved on 12.02.2019 from http://www.ncbi.nlm.nih.gov /pubmed) B. number of publications listed in Google Scholar (based on the data retrieved on 12.02.2019 from https://scholar.google.com/).

\section{Omics data}

After the completion of the Human Genome Project (1990-2003), genome sequencing technology has advanced such that the cost of 
sequencing a human-sized genome has dropped from $\$ 100$ million to below $\$ 1,000$ in addition to the dramatic reduction of the time required for sequencing. This led to the easy acquisition of the high-throughput sequencing technologies even by small-scale research laboratories. Hence, generation of huge biomedical data sets, so-called "big data" or "omics data" was inevitable and the necessity of accurately processing the data arose. To keep up with the advance in sequencing technologies, the number of available bioinformatics tools also exponentially increased resulting in a shift in our approach to solve biomedical problems ${ }^{[5]}$. When dealing with the omics data, the potential complexity increases moving from the genome and the transcriptome to the proteome ${ }^{[5]}$. It is essential to understand the biological and molecular function through analysis of the proteome for diagnosis and treatment of diseases; yet inferring proteome from the genome and transcriptome is a challenging task. Below are some examples of collaborative efforts to overcome the challenges in the data collection, analysis and interpretation steps.

\section{Collaborative efforts}

National Institutes of Health (NIH)-led Precision Medicine Initiative, with multiple other research center partners, aims to advance in individual-tailored medical care by launching All of $U s$ Research Program for collecting genetic and health data from at least one million volunteers in $\mathrm{US}^{[6]}$. Around ten thousand people enrolled to an initial pilot project on January 2018, then the national enrollment launched on May 2018 and 2022 is set for reaching the longer-term target of one million people. Expansion of cancer genomics is the near-term goal, whereas building a network of scientists to comprehensively understand health and disease based on the nationwide study is the long-term goal of the initiative ${ }^{[3]}$.

The Human Proteome Organization (HUPO) was founded in 2001, with the aim of promoting proteomics internationally to develop new technologies in the diagnosis and treatments of diseases. The Human Proteome Project (HPP), organized by HUPO and launched in 2011, is an internationally coordinated project aiming to systematically map the entire human proteome ${ }^{[7]}$. This map is planned to be generated by characterizing the whole known human genome (around 20,300 genes). Today, $89 \%$ of the project is complete (around 17,500 predicted proteins; 500 uncertain; 2,186 missing with unconfirmed/no information ${ }^{[7]}$. Upon the completion of the project, the findings are expected to improve the biology knowledge at the cellular level and help in developing medical applications that are diagnostic, predictive, therapeutic, and preventive.

Another collaborative effort in United Kingdom is the 100,000 genomes project launched in 2013 by Genomics England, which was set up by the Department of Health \& Social Care ${ }^{[8]}$. Their aim is to obtain more personalized diagnosis in cancer and rare diseases using genomic data. Participant recruitment step of the project has been completed and they will return the main findings to the participants when the analysis step finishes.

\section{Promising applications}

Oncology is a promising pioneer field in precision medicine. The solid tumors were traditionally classified by the tissue of origin but now there is a shift towards molecular classification, after being able to successfully tailor drugs based on the tested tumor's genomic or molecular profile by precision oncology ${ }^{[9-10]}$. Two main methods of precisely targeting cancer cells are the targeted therapy and immunotherapy. The first one aims the disruption of pathways essential for the cancer cell, such as growth and survival; whereas the latter one harnesses patient's own immune system to battle against cancer cells ${ }^{[11]}$. Targeted therapies require specific knowledge on the biology of the tumor and can be tailored by targeting only cancerous cells and sparing healthy cells based on this information ${ }^{[11]}$. They usually target oncogenic kinases and there are biomarker-derived personalized clinical trials that have recently been shown to be more successful than the other therapeutic options ${ }^{[10]}$. Moreover, with maximum efficacy and minimum side effects, they compromised $50 \%$ of the FDAapproved oncology drugs in $2016^{[10]}$. The pioneering targeted cancer treatment with a clinical success was the small-molecule tyrosine kinase inhibitor imatinib developed to target the constitutively active ABL oncogene for chronic myeloid leukemia $(\mathrm{CML})^{[12]}$. Some other kinase inhibitors targeting biomarkers are gefitinib for EGFR and crizotinib for ALK used in the personalized therapy in lung adenocarcinoma ${ }^{[13]}$ and trastuzumab for HER2 in breast cancer ${ }^{[14]}$. The biggest challenge of this approach has been the drug resistance acquired by patients after the therapy and as a solution, next generation kinase inhibitors were developed rapidly and a combinatorial approach was implemented ${ }^{[11]}$. One powerful example of such a case is the design of a third-generation EGFR kinase inhibitor osimertinib targeting T790M mutation and its approval by the FDA in less than 2 years ${ }^{[15]}$. Immunotherapies, the second approach, can harness the immunity of the patient through either strengthening the host response (cancer vaccines and adoptive $\mathrm{T}$ cell therapy (ACT)) or preventing immunosuppression (immune checkpoint ${ }^{[11-16]}$. Clinical trials combining targeted therapies with immunotherapies show that kinase inhibitors complement checkpoint therapy such that pathway inhibition not only affects tumor growth and angiogenesis but also tumor antigenicity and lymphocyte infiltration ${ }^{[17-18]}$. There are also other success stories of precision medicine in various diseases including Mendelian diseases (e.g. cystic fibrosis ${ }^{[19]}$ ), highly genetic diseases (e.g. epilepsy - EpiPM Consortium ${ }^{[20]}$ ) and Alzheimer's disease Alzheimer Precision Medicine Initiative (APMI) ${ }^{[21]}$; as well as in pharmacogenomics $^{[22]}$, which deals with specific responses of patients to certain doses of particular drugs.

In summary, today we are able to understand the genetic basis of diseases better, thanks to the high-throughput sequencing technologies producing accurate omics data and the bioinformatics tools being developed to efficiently interpret the highly complex data. The accurate and efficient analysis and interpretation of the omics data via bioinformatics tools, thus, leads us to tailor more precise therapies in the clinics. The collaborative efforts all around the globe pave the way for successful precision medicine applications on various diseases. There is still a long way to go but promising results keep guiding us towards a healthier future.

\section{References}

[1] "Help Me Understand Genetics page: National Library of Medicine (US). Genetics Home Reference [Internet]. Bethesda (MD): The Library; Precision Medicine," 2019 Feb 5. [Online]. Available: https://ghr.nlm.nih.gov/primer\#precisionmedicine. [Accessed February 12, 2019].

[2] N. R. Council. (2011). Toward Precision Medicine: Building a Knowledge Network for Biomedical Research 
and a New Taxonomy of Disease. Washington, DC: The National Academies Press.

[3] F. S. Collins, \& H. Varmus, "A new initiative on precision medicine." N Engl J Med, 372(9), pp. 793-795, 2015.

[4] D. Weston, \& L. Hood, "Systems biology, proteomics, and the future of health care: toward predictive, preventative, and personalized medicine." J Proteome Res, 3(2), pp. 179-196, 2004.

[5] Manzoni, D. A. Kia, J. Vandrovcova, et al., "Genome, transcriptome and proteome: the rise of omics data and their integration in biomedical sciences." Brief Bioinform, 19(2), pp. 286-302, 2018.

[6] S. Reardon, "Giant study poses DNA data-sharing dilemma." Nature, 525(7567), pp. 16-17, 2015.

[7] G. S. Omenn, L. Lane, C. M. Overall, et al., "Progress on Identifying and Characterizing the Human Proteome: 2018 Metrics from the HUPO Human Proteome Project." J Proteome Res, 2018.

[8] M. Peplow, "The 100,000 Genomes Project." BMJ, 353, pp. i1757, 2016.

[9] E. A. Ashley, "Towards precision medicine." Nat Rev Genet, 17(9), pp. 507-522, 2016.

[10] F. Cheng, H. Liang, A. J. Butte, C. Eng, \& R. Nussinov, "Personal Mutanomes Meet Modern Oncology Drug Discovery and Precision Health." Pharmacol Rev, 71(1), pp. 1-19, 2019.

[11] S. A. Dugger, A. Platt, \& D. B. Goldstein, "Drug development in the era of precision medicine." Nat Rev Drug Discov, 17(3), pp. 183-196, 2018.

[12] F. Stegmeier, M. Warmuth, W. R. Sellers, \& M. Dorsch, "Targeted cancer therapies in the twenty-first century: lessons from imatinib." Clin Pharmacol Ther, 87(5), pp. 543-552, 2010.

[13] N. I. Lindeman, P. T. Cagle, M. B. Beasley, et al., "Molecular testing guideline for selection of lung cancer patients for EGFR and ALK tyrosine kinase inhibitors: guideline from the College of American Pathologists, International Association for the Study of Lung Cancer, and Association for Molecular Pathology." J Mol Diagn, 15(4), pp. 415-453, 2013.

[14] M. M. Moasser, \& I. E. Krop, "The Evolving Landscape of HER2 Targeting in Breast Cancer." JAMA Oncol, 1(8), pp. 1154-1161, 2015.

[15] Yver, "Osimertinib (AZD9291)-a science-driven, collaborative approach to rapid drug design and development." Ann Oncol, 27(6), pp. 1165-1170, 2016.

[16] Y. Yang, "Cancer immunotherapy: harnessing the immune system to battle cancer." J Clin Invest, 125(9), pp. 3335-3337, 2015.

[17] P. Sharma, \& J. P. Allison, "Immune checkpoint targeting in cancer therapy: toward combination strategies with curative potential." Cell, 161(2), pp. 205214, 2015.

[18] P. E. Hughes, S. Caenepeel, \& L. C. Wu, "Targeted Therapy and Checkpoint Immunotherapy Combinations for the Treatment of Cancer." Trends Immunol, 37(7), pp. 462-476, 2016.

[19] Rehman, N. U. Baloch, \& I. A. Janahi, "LumacaftorIvacaftor in Patients with Cystic Fibrosis Homozygous for Phe508del CFTR." N Engl J Med, 373(18), pp. 1783, 2015.

[20] P. M. C. Epi, "A roadmap for precision medicine in the epilepsies." Lancet Neurol, 14(12), pp. 1219-1228, 2015.

[21] H. Hampel, S. E. O'Bryant, S. Durrleman, et al., "A Precision Medicine Initiative for Alzheimer's disease: the road ahead to biomarker-guided integrative disease modeling." Climacteric, 20(2), pp. 107-118, 2017.

[22] C. International Warfarin Pharmacogenetics, T. E. Klein, R. B. Altman, et al., "Estimation of the warfarin dose with clinical and pharmacogenetic data." N Engl J Med, 360(8), pp. 753-764, 2009 